\title{
Humpy LNRF-velocity profiles in accretion discs orbiting rapidly rotating Kerr black holes: a possible relation to QPOs
}

\author{
Zdeněk Stuchlík*, Petr Slaný, Gabriel Török \\ Institute of Physics, Faculty of Philosophy and Science, Silesian University in Opava, Bezručovo \\ nám. 13, CZ-74601 Opava \\ E-mail: zdenek.stuchlik@fpf.slu.cz, petr.slany@fpf.slu.cz, terek@volny.cz
}

\begin{abstract}
Change of sign of the velocity gradient (mesured with respect to locally non-rotating frames) has been found for accretion discs orbiting rapidly rotating Kerr black holes with spin $a>0.9953$ for Keplerian discs [1] and $a>0.99979$ for marginally stable thick discs [2]. Such "humpy" orbital velocity profiles occur close to but above the marginally stable circular geodesic of the black hole spacetimes. The maximal positive rate of change of the orbital velocity in terms of the proper radial distance introduces a locally defined critical frequency characterizing any processes in the disc capable to excite possible oscillations connected with the velocity hump. Comparing the "humpy frequency" related to distant observers with the epicyclic frequencies we show that in Keplerian discs orbiting extremely rapid Kerr holes $\left(1-a<10^{-4}\right)$ the ratio of the epicyclic frequencies and the humpy frequency is nearly constant, i.e., almost independent of $a$, being $\sim 3: 2$ for the radial epicyclic frequency and $\sim 11: 2$ for the vertical epicyclic frequency. For black holes with $a \sim 0.996$, i.e., when the resonant phenomena with ratio $3: 1$ between the vertical and radial epicyclic oscillations occur near the radius of the critical humpy frequency, the ratio of the radial epicyclic and the humpy frequency is $\sim 12: 1$, which is close to the ratio between high- and low- frequency QPO in X-ray systems. For $a>0.996$ the resonant orbit $r_{4: 1}$ (with the ratio $4: 1$ between the vertical and radial epicyclic oscillations) occurs in the region of the hump. Applying the model on the nearly extreme black hole candidate GRS 1915+105, we conclude that for black hole parameters $M=14.8 M_{\odot}$ and $a=0.9998$ the observed high-frequency QPOs could be related to the hump-induced oscillations in thin accretion disc, as the first two QPOs, $41 \mathrm{~Hz}$ and $67 \mathrm{~Hz}$, can be identified with the "humpy frequency" and the radial epicyclic frequency (at the same orbit). The other observed QPO-frequencies, $113 \mathrm{~Hz}$ and $166 \mathrm{~Hz}$, can be explained as the combinational ones of the "humpy" and epicyclic frequencies.
\end{abstract}

VI Microquasar Workshop: Microquasars and Beyond

September 18-22, 2006

Como, Italy

* Speaker. 


\section{Humpy orbital velocity profiles}

The Kerr metric (with the central-mass parameter $M$ and rotational parameter $a>0$ ) in standard Boyer-Lindquist (B-L) coordinates and geometrical units $(c=G=1)$ can be written in the form

$$
\mathrm{d} s^{2}=-\frac{\Sigma \Delta}{A} \mathrm{~d} t^{2}+\frac{A \sin ^{2} \theta}{\Sigma}(\mathrm{d} \varphi-\omega \mathrm{d} t)^{2}+\frac{\Sigma}{\Delta} \mathrm{d} r^{2}+\Sigma \mathrm{d} \theta^{2}
$$

where

$$
\Delta=r^{2}-2 M r+a^{2}, \quad \Sigma=r^{2}+a^{2} \cos ^{2} \theta, \quad A=\left(r^{2}+a^{2}\right)^{2}-a^{2} \Delta \sin ^{2} \theta, \quad \omega=2 a r / A,
$$

enabling to introduce a natural set of local observers - so called ZAMO (Zero Angular Momentum Observers) who, in some sense, rotate with the geometry. The related frames are called LNRF (Locally Non-Rotating Frames). Each observer carries an orthonormal tetrad of basis 1-forms [3]

$$
\begin{aligned}
\mathbf{e}^{(t)} & =\left(\frac{\Sigma \Delta}{A}\right)^{1 / 2} \mathbf{d} t, & \mathbf{e}^{(\varphi)} & =\left(\frac{A}{\Sigma}\right)^{1 / 2} \sin \theta(\mathbf{d} \varphi-\omega \mathbf{d} t), \\
\mathbf{e}^{(r)} & =\left(\frac{\Sigma}{\Delta}\right)^{1 / 2} \mathbf{d} r, & \mathbf{e}^{(\theta)} & =\Sigma^{1 / 2} \mathbf{d} \theta
\end{aligned}
$$

and, rather than describe physical quantities by their coordinate components at each point, one gives their projections onto the tetrad, i.e., their physically measured components. The local frame of any physical observer differs from the LNRF at observer's location only by a Lorentz transformation [3]. Further we put $M=1$ to obtain completely dimensionless formulae hereafter.

For matter with a 4-velocity $U^{\mu}=\left(U^{t}, 0,0, U^{\varphi}\right)$ and angular velocity $\Omega=U^{\varphi} / U^{t}$ orbiting a Kerr black hole, the orbital velocity is given by the azimuthal component of its 3-velocity in the LNRF

$$
\mathscr{V}^{(\varphi)}=\frac{U^{\mu} \mathrm{e}_{\mu}^{(\varphi)}}{U^{v} \mathrm{e}_{v}^{(t)}}=\frac{A \sin \theta}{\Sigma \sqrt{\Delta}}(\Omega-\omega) ;
$$

$\omega=-g_{t \varphi} / g_{\varphi \varphi}$, given by (1.2), is the angular velocity of the LNRF relative to distant observers.

In thin (Keplerian) discs the matter is assumed to follow nearly circular equatorial geodesics characterized by the Keplerian distribution of the angular velocity and the specific angular momentum $\ell=-U_{\varphi} / U_{t}$

$$
\Omega_{\mathrm{K}}(r ; a)=\frac{1}{\left(r^{3 / 2}+a\right)}, \quad \ell_{\mathrm{K}}(r ; a)=\frac{r^{2}-2 a r^{1 / 2}+a^{2}}{r^{3 / 2}-2 r^{1 / 2}+a} .
$$

The orbital velocity of Keplerian discs is then given by the relation

$$
\mathscr{V}_{\mathrm{K}}^{(\varphi)}(r ; a)=\frac{\left(r^{2}+a^{2}\right)^{2}-a^{2} \Delta-2 a r\left(r^{3 / 2}+a\right)}{r^{2}\left(r^{3 / 2}+a\right) \sqrt{\Delta}}
$$

and, as found by Aschenbach [1], it has a "humpy profile" in the field of rapidly rotating Kerr black holes with $a>a_{\mathrm{c}(\mathrm{K})} \doteq 0.9953$ (see Fig. 1). 

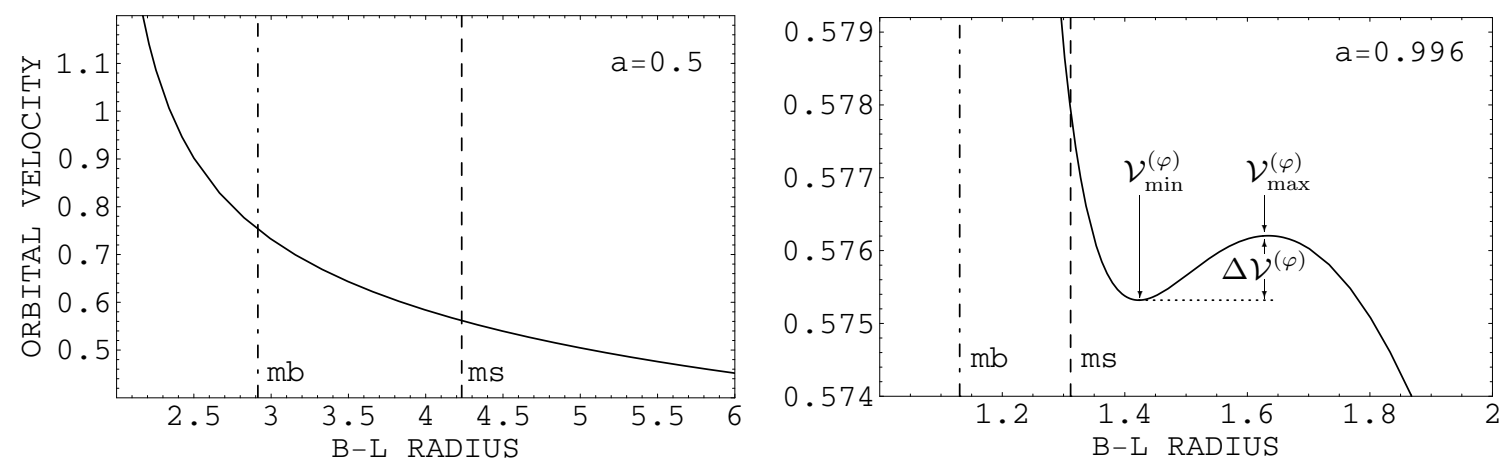

Figure 1: Radial profiles of the orbital velocity (related to the LNRF) demonstrating the existence of the velocity hump for nearly extreme Kerr black holes in the case of Keplerian discs. The dashed (dashed-dotted) line determines location of the marginally stable (marginally bound) orbit. The velocity hump $\Delta \mathscr{V}^{(\varphi)}$ reaches the maximal value for the extreme Kerr black hole being for Keplerian discs 0.07 [2].

\section{Hump-induced oscillations}

The radial gradient of the orbital velocity $\mathscr{V}^{(\varphi)}$ in the equatorial plane $(\theta=\pi / 2)$ determines the characteristic frequency of oscillations possibly induced by the LNRF-velocity hump [1,4]. To give physically correct (i.e., coordinate-independent) definition of the characteristic frequency related to local physics in the disc, we suggest to define the critical frequency by the maximum positive gradient of the orbital velocity in terms of the proper radial distance $\tilde{R}$ [5]

$$
v_{\text {crit }}^{\tilde{R}}=\left.\frac{\partial \mathscr{V}(\varphi)}{\partial \tilde{R}}\right|_{\max }, \quad \mathrm{d} \tilde{R}=\sqrt{g_{r r}} \mathrm{~d} r=\sqrt{\frac{\Sigma}{\Delta}} \mathrm{d} r .
$$

Such a locally defined frequency (confined to LNRF) should be further related to distant stationary observers by the formula (taken at the B-L coordinate $r$ corresponding to $\left(\partial \mathscr{V}^{(\varphi)} / \partial \tilde{R}\right)_{\max }$ )

$$
v_{\mathrm{h}}=v_{\infty}^{\tilde{R}}=\sqrt{-\left(g_{t t}+2 \omega g_{t \varphi}+\omega^{2} g_{\varphi \varphi}\right)} v_{\mathrm{crit}}^{\tilde{R}} .
$$

The "humpy frequency" $v_{\mathrm{h}}$ represents an upper limit on characteristic frequencies of oscillations induced by the hump of the LNRF-velocity profile, and the realistic humpy frequencies, as observed by distant observers, can be expected close to but smaller than $v_{\infty}^{\tilde{R}}$. Characteristic frequencies $v_{\text {crit }}^{\tilde{R}}, v_{\infty}^{\tilde{R}}$ are given in Table 1 for some typical values of the rotational parameter $a$.

\section{Relation to epicyclic oscillations}

It is both interesting and useful to compare the "humpy frequency" $v_{\mathrm{h}}$ with the frequencies of the orbital motion and radial and vertical epicyclic oscillations (e.g., [6, 7])

$$
v_{\mathrm{r}}^{2}=v_{\mathrm{K}}^{2}\left(1-6 r^{-1}+8 a r^{-3 / 2}-3 a^{2} r^{-2}\right), \quad v_{\mathrm{v}}^{2}=v_{\mathrm{K}}^{2}\left(1-4 a r^{-3 / 2}+3 a^{2} r^{-2}\right),
$$

where $v_{\mathrm{K}}=\Omega_{\mathrm{K}} / 2 \pi$, even if no mechanism triggering the LNRF-velocity hump excited oscillations is known exactly. Note that the resonant phenomena between the orbital and/or radial and vertical epicyclic oscillations play the crucial role in Kluźniak \& Abramowicz resonant model [8, 10], 
Table 1: Critical frequency $v_{\text {crit }}^{\tilde{R}}$, given by the maximal gradient of the orbital velocity in terms of the proper radial distance, and its value at infinity $v_{\infty}^{\tilde{R}}$ given for appropriate values of the black-hole spin in units of $\left(M / M_{\text {Sun }}\right)^{-1} \mathrm{~Hz}$. Maximum value of $v_{\infty}^{\tilde{R}}$ is bold-faced.

\begin{tabular}{clllll}
\hline $1-a$ & $4.5 \times 10^{-3}$ & $4 \times 10^{-3}$ & $3 \times 10^{-3}$ & $1 \times 10^{-3}$ & $5 \times 10^{-4}$ \\
$v_{\text {crit }}^{\tilde{\mathrm{R}}}$ & 121 & 432 & 1130 & 3061 & 3789 \\
$v_{\infty}^{\tilde{\mathrm{R}}}$ & 29 & 102 & 248 & 536 & 592 \\
\hline $1-a$ & $2 \times 10^{-4}$ & $1 \times 10^{-4}$ & $1 \times 10^{-5}$ & $1 \times 10^{-6}$ & $1 \times 10^{-9}$ \\
$v_{\text {crit }}^{\tilde{\mathrm{R}}}$ & 4352 & 4579 & 4816 & 4841 & 4844 \\
$v_{\infty}^{\mathrm{R}}$ & $\mathbf{6 0 7}$ & 603 & 590 & 588 & 588 \\
\hline
\end{tabular}

being one of the fruitful hypothesis related to the high frequency twin peak QPOs observed in $\mathrm{X}$-ray binary systems.

In thin (Keplerian) discs, the ratios of the Keplerian $\left(v_{\mathrm{K}}\right)$ and epicyclic $\left(v_{\mathrm{r}}, v_{\mathrm{v}}\right)$ frequencies to the "humpy frequency" $\left(v_{\mathrm{h}}\right)$, as well as the ratio of the epicyclic frequencies, are for the Kerr black holes with the spin parameter $1-a<10^{-4}$ almost constant and independent of $a$. Thus the $1 / M$ scaling of considered frequencies perfectly holds. The most interesting are the cases of $v_{\mathrm{r}}: v_{\mathrm{h}} \sim 3: 2$ and $v_{\mathrm{K}}: v_{\mathrm{v}} \sim 4: 1$ indicating a possibility of "doubled" resonant phenomena with the special frequency ratios. For Kerr black holes with $a \sim 0.996$ (when the resonant orbit $r_{3: 1}$ with $v_{\mathrm{v}}: v_{\mathrm{r}} \sim 3: 1$ occurs near the definition radius of the critical humpy frequency $v_{\mathrm{h}}$ ), the ratio $v_{\mathrm{r}}: v_{\mathrm{h}} \sim$ $12: 1$, which is close to the PBK correlation [9] between the observed high- and low- frequency QPO. However, the PBK correlation extends through large scale of systems having often spacetime conditions preclusive the existence of humpy induced oscillations. Therefore, the coincidence occuring for black holes with $a \sim 0.996$ is rather accidental. For $a>0.996$ the resonant orbit $r_{4: 1}$ (with $v_{\mathrm{v}}: v_{\mathrm{r}} \sim 4: 1$ ) occurs in the region of the hump.

\section{GRS 1915+105}

The model of hump-induced oscillations seems to be applicable to the nearly extreme blackhole candidate - the microquasar GRS 1915+105 revealing four HFQPOs with the frequencies $41 \mathrm{~Hz}, 67 \mathrm{~Hz}, 113 \mathrm{~Hz}$ and $166 \mathrm{~Hz}$ [11]. The last two frequencies meet the "magic ratio" $3: 2$ typical for all known microquasars in which the twin peak HFQPOs were observed, (XTE 1550-564, GRO 1655-40, GRS 1915+105, H 1743-322), see, e.g. [10], indicating the role of some resonant phenomena in accretion disc around black hole. Taking into account the mass and spin estimates for the central black hole, $M_{\mathrm{BH}}=14.0 \pm 4.4 M_{\odot}, a>0.98$ [11], we conclude that for $M=14.8 M_{\odot}$ and $a=0.9998$ the first two frequencies, $41 \mathrm{~Hz}$ and $67 \mathrm{~Hz}$, could be identified with the "humpy frequency" $v_{\mathrm{h}}$ and the radial epicyclic frequency $v_{\mathrm{r}}$ (on the orbit where the proper radial gradient of the orbital velocity $\mathscr{V}^{(\varphi)}$ is maximal), respectively. Note that till now perhaps no other model of HFQPOs has tried to interpret this pair of frequencies. Remarkably, if we take into account the forced resonance phenomena between the hump-induced and epicyclic oscillations, and the combinational frequencies, also the next pair of frequencies, $113 \mathrm{~Hz}$ and $166 \mathrm{~Hz}$, being measured with uncertainties $\pm 5 \mathrm{~Hz}$, can be explained in terms of the hump-induced oscillations, as these 
frequencies could be given by the forced resonance in the beat frequency $v_{\mathrm{h}}+v_{\mathrm{r}}=41+67=$ $108 \mathrm{~Hz}$ and the difference beat frequency $v_{\mathrm{v}}-v_{\mathrm{r}}=237-67=170 \mathrm{~Hz}$, where $v_{\mathrm{v}}$ is the vertical epicyclic frequency determined at the same orbit as the previous ones. Thus all four observed frequencies in GRS 1915+105 could be emitted from the narrow region of the velocity hump, and closely related to the hump-induced oscillations in a thin accretion disc assumed to be present in GRS $1915+105$.

\section{Acknowledgments}

The work has been done as a part of the research project MSM 4781305903.

\section{References}

[1] B. Aschenbach, Measuring mass and angular momentum of black holes with high-frequency quasi-periodic oscillations, Astron. Astrophys. 425, 1075 (2004) [astro-ph/ 040654 5].

[2] Z. Stuchlík, P. Slaný, G. Török and M. A. Abramowicz, Aschenbach effect: Unexpected topology changes in motion of particles and fluids orbiting rapidly rotating Kerr black holes, Phys. Rev. D 71, 024037 (2005) [gr-qc/ 0411091 ].

[3] J. M. Bardeen, W. H. Press and S. A. Teukolsky, Rotating black holes: locally nonrotating frames, energy extraction, and scalar synchrotron radiation, Astrophys. J. 178, 347 (1972).

[4] B. Aschenbach, Mass and Angular Momentum of Black Holes: An Overlooked Effect of General Relativity Applied to the Galactic Center Black Hole Sgr A*, (2006) [astro-ph / 603193 ].

[5] Z. Stuchlík, P. Slaný and G. Török, Orbital velocity gradient inversion of marginally stable thick discs in locally non-rotating frames, in Proceedings of RAGtime 4/5: Workshops on black holes and neutron stars, Opava, 14-16/13-15 Oct 2002/03, S. Hledík and Z. Stuchlík (eds.), Silesian University in Opava (2004).

[6] A. N. Aliev and D. V. Galtsov, Radiation from relativistic particles in non-geodesic motion in a strong gravitational field, Gen. Relativ. Grav. 13, 899 (1981).

[7] M. A. Nowak and D. E. Lehr, Stable oscillations of black hole accretion discs, in Theory of Black Hole Accretion Disks, M. A. Abramowicz, G. Björnsson and J. E. Pringle (eds.), Cambridge University Press, Cambridge (1998).

[8] W. Kluźniak and M. A. Abramowicz, Strong field gravity and orbital resonance in black holes and neutron stars — kHz quasi periodic oscillations, Acta Phys. Polonica B 32, 3605 (2001).

[9] D. Psaltis, T. Belloni, M. van der Klis, Correlations in Quasi-periodic Oscillation and Noise Frequencies among Neutron Star and Black Hole X-Ray Binaries, Astrophys. J., 520, 262 (1999) [astro-ph/9902130].

[10] G. Török, M. A. Abramowicz, W. Kluźniak and Z. Stuchlík, The orbital resonance model for twin peak $\mathrm{kHz}$ quasi periodic oscillations in microquasars, Astron. Astrophys. 436, 1 (2005) [astro-ph/0401464].

[11] J. E. McClintock et al., The Spin of the Near-Extreme Kerr Black Hole GRS 1915+105, accepted for publication in Astrophys. J., (2006) [astro-ph/0606076]. 